\title{
Circadian rhythms in RA
}

\section{Cutolo, B Seriolo, C Craviotto, C Pizzorni, A Sulli}

\section{Possible roles of cortisol and melatonin}

$\mathrm{t}$ is well known that some clinical signs and symptoms of rheumatoid arthritis (RA) vary within a day and between days, and the morning stiffness seen in patients with RA has become one of the diagnostic criteria of the disease (fig 1).

Among the clinical signs of joint inflammation in patients with RA, the intensity of pain changes consistently as a function of the hours of the day: pain is greater after waking up in the morning than in the afternoon or evening. ${ }^{2}$ In patients with RA circadian variations are also found in joint swelling and finger size and these symptoms are in phase with the circadian rhythm of pain. The RA rhythms differ in phase by about 12 hours from the circadian changes of left and right hand grip strength: a greater grip strength is seen when joint circumferences and the subjective ratings of stiffness and pain are least and vice versa. "Clinical signs and symptoms in
RA depend on the time of day"

Therefore, clinical signs and symptoms in RA show a rhythm that seems driven by a biological clock.

\section{Biological rhythms in experimental inflammation}

Biological rhythms have been seen in different models of inflammation, and maximal inflammation occurred during the activity period of the animals - that is, between midnight and $800 \mathrm{am} .^{4}$

Biological rhythms with a periodicity longer than 24 hours have also been detected, and a circaseptan rhythm (almost seven days) of paw oedema, over a period of 30 days, was observed, with and vice versa. ${ }^{4}{ }^{6}$ peak of inflammation every 6-7 days. ${ }^{5}$ Furthermore, circannual variations have been identified in different models of inflammation showing that maximal articular oedema was significantly larger in spring and lowest in winter. ${ }^{6}$ A time dependent change of blood flow at the inflammatory site may also explain the circadian variations in experimental oedema; some studies in rat models showed that the blood flow was greater in the night and lower in the morning. ${ }^{2}$

The mechanisms of the time dependent variations of the inflammatory reaction are complex and include several systems of mediators (that is, histamine, bradikinin, prostaglandin, and mainly, pro- and anti-inflammatory cytokine production). However, the circadian changes in the metabolism or secretion of endogenous corticosteroids are certainly implicated in the time dependent changes seen during the inflammatory response. This assumption is supported by data showing that adrenalectomy abolished the circadian variation in the rate of formation of experimental oedema and that this was restored by hydrocortisone administration. ${ }^{7}$ Other data indicate that the inflammatory response, produced by different agents, was in phase opposition to the cortisol rhythm: less oedema was obtained when the plasma cortisol levels were higher

More recently, melatonin (MLT), another circadian hormone that is the secretory product of the pineal gland, has been found to be implicated in the time dependent inflammatory reaction, with effects opposite to those of cortisol. ${ }^{8}$ In several species, pinealectomy or any other experimental procedure

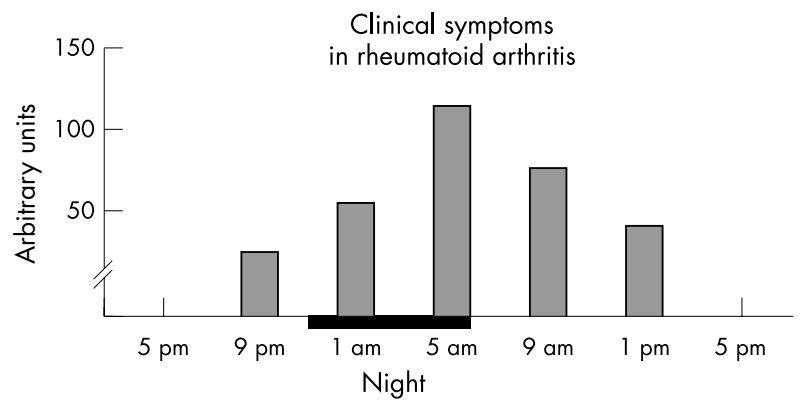

Figure 1 Clinical signs and symptoms of articular inflammation in patients with RA change consistently as a function of the hours of the day: pain and joint stiffness are greater after waking up in the morning than in the afternoon or evening. that inhibits MLT synthesis and secretion induces a state of immunodepression, which is counteracted by MLT replacement. ${ }^{9-11}$ In general, MLT displays an immunoenhancing effect. ${ }^{12}$ MLT can activate $\mathrm{T}$ lymphocytes, monocytes, NK cells, and even neutrophils, activates antibody dependent cellular cytotoxicity, and enhances antibody responses in vivo. ${ }^{10}$ In animal models, as well as in human and in vitro experiments, MLT enhances inflammatory cytokine and nitric oxide production. ${ }^{13}{ }^{14}$ In addition, the in vitro effects exerted by glucocorticoids on the immune function seem modulated by MLT in physiological to pharmacological concentrations. ${ }^{15}$

\section{Cortisol and melatonin regulate} circadian cytokine production

In adult primates, only visible light (400-700 nm) is received by the retina. This photic energy is then transduced and delivered to the visual cortex and, by an alternative pathway, to the suprachiasmatic nucleus, the hypothalamic region that directs circadian rhythms. Visible light exposure also modulates the pituitary and pineal glands, leading to neuroendocrine changes. MLT, norepinephrine, and acetylcholine decrease with light activation, whereas cortisol, serotonin, $\gamma$-aminobutyric acid, and dopamine levels increase. ${ }^{16}$

Therefore, ocular light seems to be the predominant modulator and major determinant of circadian rhythm for many neurohormones, with cortisol and MLT showing an opposite response to the light. The light conditions in the early morning have a strong impact on the morning cortisol peak, whereas direct inhibitory effects of light on pineal activity may contribute to phasing of the onset and termination of MLT production in a strictly nocturnal pattern. ${ }^{917}$

\section{"Melatonin counteracts the effects of cortisol"}

Recently, a diurnal rhythmicity in healthy humans between cellular (Thl type) or humoral (Th2 type) immune responses has been found and related to immunomodulatory actions of cortisol and MLT (fig 2). ${ }^{18}$ In particular, the production of the interferon $\gamma$ (IFN $\gamma$; type 1) and interleukin 10 (IL10; type 2) in human whole blood stimulated with lipopolysaccharide or tetanus, as well as the IFN $\gamma / \mathrm{IL} 10$ ratio, exhibited a significant diurnal rhythmicity. The IFN $\gamma /$ ILI0 ratio peaked during the early morning and correlated negatively with plasma cortisol and positively with plasma MLT; the IFN $\gamma / \mathrm{IL} 10$ ratio decreased by $>70 \%$ after the administration of oral cortisone acetate $(25 \mathrm{mg})$. Therefore, these findings support the concept that plasma cortisol and possibly MLT seem to 


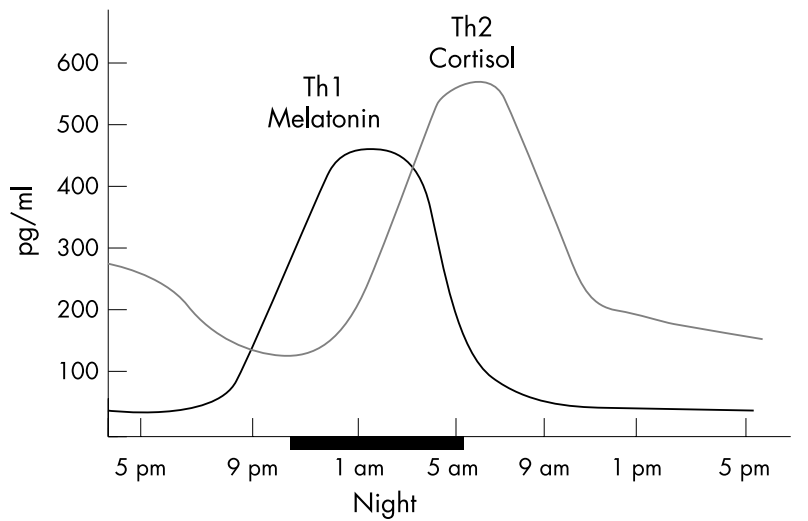

Figure 2 A diurnal rhythmicity in healthy humans between cellular (Th 1 type) or humoral (Th2 type) immune responses has been found and related to the immunomodulatory effects exerted by cortisol and melatonin, respectively.

regulate diurnal variations of cytokine production.

In normal subjects, MLT peaks at about 3 am, whereas cortisol peaks at about $4 \mathrm{am} .{ }^{19}$ Interestingly, ILl, IL6, and soluble IL2 receptors peak at $1-4$ am and are low throughout the day. ${ }^{20}$ MLT stimulates ILl and IFN $\gamma$ production by human monocytes, and serum IL2 increases during the night concomitantly with the rise in MLT; MLT also seems to enhance IL2 immunomodulating effects. ${ }^{21-23}$ In addition, MLT increases the production of IL12 and nitric oxide by cultured human synovial macrophages, enhances IL2, IL6, and IFN $\gamma$ production by human circulating CD4+ lymphocytes, and up regulates the level of gene expression of tumour necrosis factor $\alpha$ $(\mathrm{TNF} \alpha)$ and macrophage-colony stimulating factor. ${ }^{14} 2425$

On the contrary, cortisol was found to be negatively correlated with the IFN $\gamma /$ IL10 ratio, and cortisone administration markedly reduced this ratio with a clear causal relationship. ${ }^{18}$ Furthermore, similarly to IFN $\gamma$ and ILl, TNF $\alpha$ and IL12 also exhibit distinct diurnal rhythms that peak in the early morning, and these changes are inversely related to the rhythm of plasma cortisol. ${ }^{26}$

In conclusion, because IFN $\gamma$ and ILI0 might be considered markers of cellular (type 1) and humoral (type 2) immunity, respectively, these circadian studies suggest that there is a bias towards cellular immunity during the night and early morning (peak of MLT) when the IFN $\gamma /$ IL10 ratio is high and, conversely, a relative bias towards humoral (type 2) immunity during the rest of the day (cortisol effects) (fig 2). ${ }^{27}$

\section{Cortisol and melatonin effects on circadian rhythms in rheumatoid arthritis}

The inflammatory cytokines (that is, IL6, ILl, TNF $\alpha$ ), as soluble products of the activated immune system, stimulate in the central nervous system, the production of corticotrophin releasing hormone
$(\mathrm{CRH})$ in the hypothalamus: $\mathrm{CRH}$ release leads to pituitary production of adrenocorticotrophic hormone (ACTH), followed by glucocorticoid secretion by the adrenal cortex..$^{28} 29$

These components constitute the hypothalamic-pituitary-adrenocortical axis (HPA).

Recently, intact ACTH secretion, but impaired cortisol response in patients with active RA has been described and this observation was consistent with a relative adrenal glucocorticoid insufficiency, the latter already suggested 40 years earlier. ${ }^{30} 31$ Increased HPA axis function is a normal response to the stress of inflammation and may be mediated by central and peripheral actions of circulating cytokines.

Besides ILl and TNF $\alpha$, IL6 seems to be a major factor mediating interactions between the activated immune system and both the anterior pituitary cells (central) and the adrenal (peripheral) steroidogenesis. However, recent studies in patients with RA have shown that the overall activity of the HPA axis remains inappropriately normal (or low) and is apparently insufficient to inhibit ongoing inflammation, at least in patients with early untreated arthritis. ${ }^{32} 33$

In particular, in the early morning hours, an earlier surge of plasma ACTH and cortisol was seen in patients with RA, who at the same time had significantly increased IL6 levels and a pronounced circadian variation of plasma levels in comparison with healthy subjects. ${ }^{34}$

In addition, in the patients with RA, a positive temporal correlation was found between plasma IL6 levels and ACTH/ cortisol, with raised levels of IL6 before the increases of ACTH and cortisol by one and two hours, respectively. ${ }^{34}$ In the same patients, a negative effect of cortisol upon IL6 was found, exerted with a delay of five hours, confirming that the HPA axis in RA is apparently insufficient to inhibit ongoing inflammation.
More recently, another study showed a significantly altered secretion of adrenal androgens in premenopausal patients with RA not treated with glucocorticoids. ${ }^{35}$ In the same study, low plasma levels of the adrenal androgen dehydroepiandrosterone (DHEA) and its sulphate metabolite were found to be significantly correlated with early morning low cortisol concentrations and high basal levels of IL6 in patients with RA. ${ }^{35}$ Early morning IL6 peak values were recently found to be higher in patients with RA than in controls, and significantly correlated with morning $\mathrm{C}$ reactive protein levels and Ritchie's index. ${ }^{36}$ The observation of reduced DHEA production, combined with normal cortisol production during oCRH and ACTH testing, further support the concept of the presence of an adrenal hypofunction in patients with active RA. ${ }^{37}$

IL6 had a strong effect on steroid release and may be one of the factors controlling the long term adrenal response to stress, because this cytokine can act synergistically with ACTH on the adrenal cells to stimulate the release of corticosterone..$^{38}$ Therefore, the reduced cortisol and adrenal androgen secretion, seen during testing in patients with RA not treated with glucocorticoids, should be clearly regarded as a "relative adrenal insufficiency" in a sustained inflammatory process, as shown by the high IL6 levels. ${ }^{40}$

\section{"Low dose prednisolone has more effect on RA at 2 am than at 7.30 $\mathrm{am}^{\prime \prime}$}

In a very recent investigation on salivary cortisol levels in patients with recent onset RA, it was found that afternoon cortisol concentrations in patients with high disease activity did not drop, as did the cortisol levels in healthy controls and patients with RA with low disease activity. ${ }^{41}$ These findings further indicate that activation of the HPA axis in RA is evident, but insufficient. The lower than required production of cortisol supports the efficacy of low dose glucocorticoid "replacement therapy" in patients with RA. ${ }^{42}$ From recent studies, it seem that administration of low doses of glucocorticoid with a rather short biological half life may improve acute RA symptoms if the administration precedes the period of circadian flare in inflammatory activity, as defined by enhanced IL6 synthesis. ${ }^{43}$ In fact, administration of low doses of prednisolone at 200 am was found to induce significantly more favourable effects on RA clinical symptoms than administration at $730 \mathrm{am} .{ }^{43}$ Therefore, timing of low dose glucocorticoid administration should be adapted to the biological rhythms of the inflammatory process in RA (that is, rhythms of inflammatory cytokines such as IL6). ${ }^{44}$ 


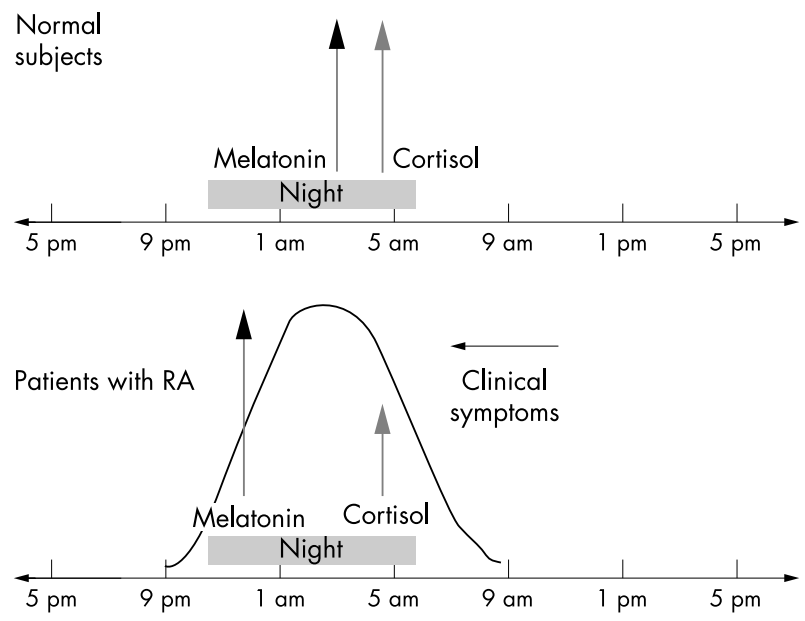

Figure 3 A lower than expected cortisol secretion as seen during testing in patients with RA, should be clearly regarded as a "relative adrenal insufficiency" in the setting of a sustained inflammatory process (that is, high IL6 serum levels).

Very recent studies have evaluated MLT levels in patients with RA, together with an analysis of circadian variations. ${ }^{45}$ Interestingly, MLT serum levels at $8 \mathrm{pm}$ and 8 am were found to be significantly higher in patients with RA than in controls $(p<0.05)$. The differences were greater in the older patients with RA (age $>60$ years) than in the younger ones. Both in patients with RA and healthy subjects, MLT levels progressively increased from $8 \mathrm{pm}$ to the early hours of the morning, but reached a peak in patients with RA at $12 \mathrm{pm}$, at least two hours before that in controls (fig 3). Subsequently, in patients with RA, MLT concentrations reached a plateau, lasting 2-3 hours; this effect was not evident in the controls. After $2 \mathrm{am}$ MLT levels decreased similarly both in patients with RA and healthy subjects. The results of the study confirm the existence of a nocturnal rhythm of MLT also in patients with RA. However, the peak appears earlier in the night and lasts longer in the early morning than in healthy controls. ${ }^{45}$

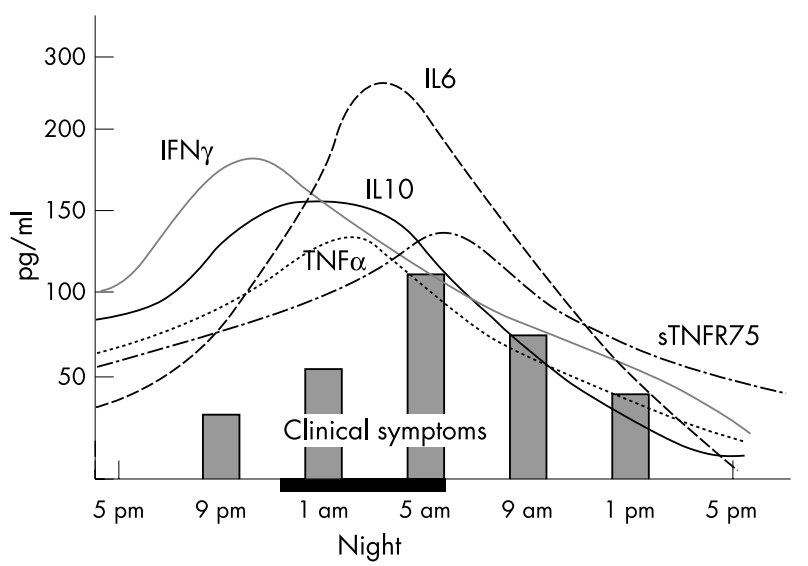

Figure $4 \mathrm{IFN} \gamma, \mathrm{IL} 2$, as well as ILL, IL6, IL12, and TNF $\alpha$ production (Th 1 cytokines) reach a peak during the night and early morning, when MLT serum levels are highest and plasma cortisol levels the lowest. Therefore, MLT may be implicated in a more active inflammatory response during the night, and the clinical symptoms follow this rhythm in patients with RA. with RA. The clinical symptoms show a circadian variation, with joint stiffness and pain being more prominent in the early morning.

Consistently, human proinflammatory cytokine production exhibits a diurnal rhythmicity, with peak levels during the night and early morning when plasma cortisol level is lowest and MLT level is highest.

In particular, Thl type cytokines that are mainly involved in RA, significantly increase, with an earlier peak in relation to altered peaking of both cortisol and MLT. An inappropriate low secretion of cortisol is a typical feature of the inflammatory disease in patients with RA. On the contrary, the nocturnal rhythm of MLT shows an earlier peak and a longer peak duration in the early morning in patients with RA than in normal subjects.

Therefore, an imbalance between antiinflammatory effects exerted by cortisol and proinflammatory effects exerted by MLT during the night seems evident in patients with RA and suggests that this imbalance may have a crucial pathogenic role in RA, and may also drive the circadian rhythm of the clinical symptoms (that is, morning stiffness and pain). ${ }^{49}$

The relief of the clinical symptoms in RA, including the anti-inflammatory efficacy exerted by morning low dose corticosteroid "replacement" therapy, seems to confirm the importance of these circadian mechanisms. Inhibitors of MLT synthesis or MLT antagonists may in the future be considered as further possible therapeutic tools, at least in severe cases of RA.

Ann Rheum Dis 2003;62:593-596

\section{Authors' affiliations}

M Cutolo, B Seriolo, C Craviotto, C Pizzorni, A Sulli, Research Laboratory and Division of Rheumatology, Department of Internal Medicine University of Genova, Viale Benedetto XV, 6 16132 Genova, Italy

Correspondence to: Professor M Cutolo; mcutolo@unige.it

\section{REFERENCES}

1 Kowanko IC, Knapp MS, Pownall R, Swannel AJ. Domiciliary self-measurement in rheumatoid arthritis and in the demonstration of circadian rythmicity. Ann Rheum Dis 1982:41:453-5

2 Labrecque G, Bureau JP, Reinberg AE. Biological rhythms in the inflammatory response and in the effects of non-steroidal anti-inflammatory drugs. Pharmac Ther 1995;66:285-300

3 Harkness JAL, Richter MB, Panayi GS, Van Depete K, Unger K, Pownall R, et al. Circadian variations in disease activity in rheumatoid arthritis. BM 1982;284:551-4

4 Soliman KFA, Soliman MR, Owasogo JO, Walker CA. Diurnal variation in the phlogistic response of rats to inflammatory agents. J Pharm Pharmac 1983;35:388-9.

5 Muir N, Pownall R. Identification of a seven day biological cycle in rat. J Pharm Pharmac 1983;36:185-8. 
6 Loubaris N, Cros G, Serrano JJ, Katz S, Bouchard S. Circadian and circannual variations of the carrageenan inflammatory effects in the rat. Life Sci 1983:32:1349-53.

7 Labreque G, Durè F, Bèlanger PM. Circadian variation of carrageenan paw edema in the rat. Life Sci 1981;28:1337-43.

8 Maestroni GJ, Conti A, Pierpaoli W. Role of the pineal gland in immunity: diurnal synthesis and release of melatonin modulates the antibody response and antagonizes the immunosuppressive effects of corticosterone. J Neuroimmunol 1986;13:19-26.

9 Reiter RJ. Pineal melatonin: cell biology of its synthesis and of its physiological interactions. Endocrinol Rev 1991;12:151-80.

10 Maestroni GJ. The immuneneuroendocrine role of melatonin. J Pineal Res 1993;14:1-10.

11 Maestroni GJ. T-helper 2 lymphocytes as a peripheral target of melatonin. J Pineal Res 1995; 18:84-9.

12 Morrey KM, McLachlan JA, Serkin CD, Bakouche $O$. Activation of human monocytes by the pineal hormone melatonin. J Immunol 1994:153:2671-80.

13 Esquifino Al, Cardinali DP. Effects of melatonin on 24-h rhythms of neuroendocrine and immune changes in Freund's adjuvant-induced arthritis. Neuroendocrinol Lett 1999:20:163-6.

14 Garcia-Mauriño S, Gonzalez-Haba MG, Calvo JR, Rafii-El-Idrissi M, Sanchez-Margalet V, Goberna R, et al. Melatonin enhances IL-2, IL- 6 and IFN- $\gamma$ production by human circulating CD4+ cells. J Immuno 1997; 159:574-81.

15 Rogers N, van den Heuvel C, Dawson D. Effect of melatonin and corticosteroid on in vitro cellular immune function in humans. J Pineal Res 1997;22:75-80.

16 Roberts JE. Light and immunomodulation. Ann NY Acad Sci 2000;917:435-45.

17 Scheer FA, Buijs RM. Light affects morning salivary cortisol in humans. J Clin Endocrinol Metab 1999:84:3395-8.

18 Petrovsky N, McNair P, Harrison L. Diurnal rhythms of pro-inflammatory cytokines: regulation by plasma cortisol and therapeutic implications. Cytokine 1998;10:307-12.

19 Miyatake A, Morimoto Y, Uishi T. Circadian rhythm of serum testosterona and its relation to sleep: comparison with the variation in serum luteinizing hormone, prolactin, and cortisol in normal men. J Clin Endocrinol Metab 1980:51:1365-71.

20 Gudewill S, Pollmacher I, Vadder $\mathrm{H}$, Schreiber W, Fassbender K, Holsboer F. Noctural plasma levels of cytokines in healthy men. Eur Arch Psychiatry Clin Neurosci 1992:242:53-6.

21 Morrey KM, McLachlan JA, Serkin CD, Bakouche $O$. Activation of human monocytes by pineal hormone melatonin. J Immunol 1994; 153:2671-80.

22 Lissoni P, Rovelli F, Brivio F, Brivio O Fumagalli L. Circadian secretions of IL-2, IL-12, IL-6 and IL-10 in relation to light/dark rhythm of the pineal hormone melatonin in healthy subjects. Nat Immun 1998;16:1-5.
23 Lissoni P. Modulation of anticancer cytokines IL-2 and IL-12 by melatonin and the othe pineal indoles 5-methoxytryptamine and 5-methoxytryptolol in the treatment of human neoplasms. Ann NY Acad Sci

2000:917:560-7.

24 Cutolo M, Villaggio B, Candido F, Valenti S, Giusti $M$, Felli $L$, et al. Melatonin influences IL-1 2 and nitric oxide production by primary cultures of rheumatoid synovial macrophages and THP-1 cells. Ann NY Acad Sci 1999;876:246-55

25 Liu F, Ng TB, Fung MC. Pineal indoles stimulates the gene expression of immunomodulating cytokines. J Neural Transm 2001;108:397-405

26 Petrovsky N, Harrison LC. The chronobiology of human cytokine production. Int Rev Immunol 1998; 16:635-49.

27 Petrovsky N. Towards a unified model of neuroendocrine-immune interaction Immuno Cell Biol 2001;78:350-7

28 Masi AT, Feigenbaum SL, Chatteron RT, Cutolo M. Integrated Hormonal-immunological-vascular ("H-I-V" Triad) system interactions in rheumatic diseases. Clin Exp Rheumatol 1995;13:203-16.

29 Chrousos GP. The hypothalamic-pituitary-adrenal axis and immune-mediated inflammation. N Engl J Med 1995;332:1351-62.

30 Gudbjörnsson B, Skogseid B, Öberg B Wide L, Hällgren R. Intact adrenocorticotropic hormone secretion but impaired cortisol response in patients with active rheumatoid arthritis. Effect of glucocorticoids. J Rheumatol 1996;23:596-602.

31 West HF. Corticosteroid metabolism and rheumatoid arthritis. Ann Rheum Dis 1993; 16:173-81.

32 Masi AT, Chrousos GP. Hypothalamic-pituitary-adrenal-glucocorticoid axis function in rheumatoid arthritis. $J$ Rheumatol 1996;23:577-81

33 Straub RH, Cutolo M. Impact of the hypothalamic-pituitary-adrenal/gonadal axes and the peripheral nervous system in rheumatoid arthritis: a systemic pathogenic view point. Arthritis Rheum 2001;44:493-507.

34 Crofford LJ, Kalogeras KT, Mastorakos G Magiakou MA, Wells J, Kanik KS, et al. Circadian relationships between interleukin (IL)-6 and hypothalamic-pituitary-adrenal axis hormones: failure of IL-6 to cause sustained hypercortisolism in patients with early untreated rheumatoid arthritis. J Clin Endocrinol Metab 1997;82:1279-83.

35 Cutolo M, Foppiani L, Prete C Ballarino P, Sulli A, Villaggio B, et al. Hypothalamic-pituitary-adrenocortical axis in premenopausal rheumatoid arthritis patients: not treated with glucocorticoids. J Rheumato 1999;26:282-8.

36 Arvidson GN, Gudbjørsson G, Elfman L, Rydén AC, Titterman TH, Hällgren R. Circadian rhythm of serum interleukin- 6 in rheumatoid arthritis. Ann Rheum Dis 1994;53:521-4.
37 Templ E, Koeller M, Riedl M, Wagner O, Graninger W, Luger A. Anterior pituitary function in patients with newly diagnosed rheumatoid arthritis. Br J Rheumato 1996:35:350-6

38 Mastorakos G, Chrousos GP, Weber JS Recombinant interleukin-6 activates the hypothalamic-pituitary-adrenal axis in human. J Clin Endocr Metab 1993;77:1690-4

39 Ehrhart-Bornstein M, Hinson JP, Bornstein SR, Scherbaum WA, Vinson GP. Intraadrenal interactions in the regulation of adrenocortical steroidogenesis. Endocrinol Rev 1998; 19:101-3.

40 Straub RH, Paimela L, Peltomaa R, Schölmerich J, Leirisalo-Repo M. Inadequately low serum levels of steroid hormones in relation to IL-6 and TNF in untreated patients with early rheumatoid arthritis and reactive arthritis. Arthritis Rheum 2002;46:654-62.

41 Dekkers JK, Greenen R, Godaert GLR, van Doornen LJP, Bijlsma LWJ. Diurnal rhythm of salivary cortisol levels in patients with recent-onset rheumatoid arthritis. Arthitis Rheum 2000:43:465-7.

42 van Everdingen AA, Jacobs JW, Siewertsz Van Reesema DR, Bijlsma JW. Low-dose prednisone therapy for patients with early active rheumatoid arthritis: clinical efficacy, disease-modifying properties, and side effects: a randomized, double-blind, placebo-controlled clinical trial. Ann Intern Med 2002;136:1-12.

43 Arvidson NG, Gudbjorsson B, Larsson A, Hallgren R. The timing of glucocorticoid administration in rheumatoid arthritis. Ann Rheum Dis 1997:56:27-31.

44 Buttgereit F, Kirwan J, Boers M, Burmester GR, Cutolo $M$, Jacobs J, et al. Standardised nomenclature for glucocorticoid dosage and glucocorticoid treatment regimens: current questions and tentative answers in rheumatology. Ann Rheum Dis 2002;61:718-22.

45 Sulli A, Maestroni GJM, Villaggio B, Hertens E, Craviotto $C$, Cutolo $M$, et al. Melatonin serum levels in rheumatoid arthritis. Ann NY Acad Sci 2002;966:276-83.

46 Schulze-Koops H, Lipsky PE, Kavanaugh AF, Davis LS. Elevated Th 1-or ThO-like cytokine mRNA in peripheral circulation of patients with rheumatoid arthritis. J Immunol 1995; 155:5029-37

47 Maestroni GJM, Sulli A, Pizzorni C, Villaggio B, Cutolo M. Melatonin in rheumatoid arthitis: synovial macrophages show melatonin receptors. Ann NY Acad Sci 2002;966:271-5

48 Maestroni GJM, Sulli A, Pizzorni C, Villaggio $B$, Cutolo M. Melatonin in rheumatoid arthritis: a disease promoting and modulating hormone? Clin Exp Rheumato 2002;20:872-3

49 Cutolo M. Solar lights effects on onset/relapses and circannual/circadian symptomology in rheumatoid arthritis. Clin Exp Rheumatol 2003;21:148-50. 